\title{
LITERACIA DIGITAL E LITERACIA INFORMACIONAL: breve análise dos conceitos a partir de uma revisão sistemática de literatura
}

\author{
Altina Ramos ${ }^{*}$ \\ Paulo Faria $^{* *}$
}

\section{Resumo}

Os conceitos de literacia digital e literacia informacional ora surgem como quase sinónimos, para alguns autores, ora com definições distintas, para outros. O que uns consideram literacia digital é por outros denominado literacia informacional. Neste artigo propomo-nos analisar ambos os conceitos a partir dos resultados de uma revisão sistemática de literatura realizada nas bases de dados ISI (ISI Web of Knowledge) e ERIC (Education Resources Information Center). Assim, apresentamos a) o processo e o produto da revisão sistemática com apoio do EndNote para cada um dos conceitos; b) a análise dos dados com recurso a NVivo c) a conclusão acerca do atual estado da arte quanto ao conceito de literacia digital e de literacia informacional.

Palavras-chave: Digital literacy. Informational literacy. Revisão sistemática. Investigação exploratória.

\section{DIGITAL LITERACY AND INFORMATION LITERACY: a brief analysis of the concepts based on a systematic review of the literature}

\begin{abstract}
The concepts of digital literacy and information literacy sometimes emerge as almost synonymous, for some authors, sometimes with different definitions, for others. Which some consider digital literacy, others call informational literacy. In this paper we will consider both of these concepts based on the results of a systematic literature review carried out in the ISI databases (ISI Web of Knowledge) and ERIC(Education Resources Information Center). Thus, we present a) the process and results of systematic review with the support of EndNote for each of the concepts; b) data analysis using NVivo; c) the conclusion concerning the actual state of the art regarding the concepts of digital literacy and information literacy.
\end{abstract}

Keywords: Digital literacy. Information literacy. Systematic review. Exploratory research.

\footnotetext{
* Doutora em Estudos da Criança e Professora da Universidade do Minho - Portugal. E-mail: altina@ie.uminho.pt

** Doutor em Ciências da Educação e Professor da Universidade do Minho - Portugal. E-mail: paulofaria@ie.uminho.pt
} 


\section{Introdução}

Segundo nos foi possível apurar, o dicionário da Academia das Ciências de Lisboa (2001) é o primeiro, no português europeu, que contempla uma entrada para o termo literacia. Dos dois significados que apresenta, o primeiro define literacia como capacidade de ler e escrever em oposição a iliteracia; o segundo, como condição ou estado de pessoa instruída.

No panorama anglo-saxónico, a realidade já é bem diferente. Neste contexto, o conceito literacia tem suscitado um profundo interesse, tendo levado já em 1995 à publicação de um dicionário dedicado exclusivamente ao tema literacia, denominado The literacy dictionary. The vocabular of reading and writing, editado por Harris e Hodges; em 1999 surge uma nova versão abreviada deste dicionário. É, justamente, desta última publicação que transcrevemos a entrada literacy, assinada por Venezky (1995) e definida assim:

Literacy, therefore, requires active, autonomous engagement with print and stresses the role of the individual in generating as well as receiving and assigning independent interpretation to messages. By extension of the basic competence implied by literacy, computer literacy, cultural literacy, economic literacy, and so forth have evolved as designations of minimal competence required in these areas. (HARRIS \& HODGES, 1995, p. 19) ${ }^{1}$

Pressupõe-se, então, de que a referência a literacia(s) é entendida enquanto competência em continuum desenvolvimento inerente ao indivíduo, como de resto também foi reconhecido no Programme from International Student Assessmente (PISA, 2000), logo na introdução do documento Assessment of reading, mathematical and scientific literacy.

O relevo público do termo e o crescente interesse científico por estes conceitos têm suscitado uma abordagem paradoxal, por vezes contraditória. Porém, neste texto, interessanos compreender como são entendidas as expressões literacia digital e literacia informacional. Este estudo exploratório tem como intuito dar a conhecer, clarificar as expressões nomeadas e tentar delimitar as fronteiras entre ambos os conceitos. Para isso, optamos por realizar uma revisão sistemática de literatura, metodologia muito frequente em algumas áreas do conhecimento, particularmente no domínio das Ciências Médicas.

Assim, e face à amplitude semântica dos conceitos e à sua plurissignificação,

\footnotetext{
${ }^{1}$ As organizadoras do Dossiê optaram por inserir em nota de rodapé a tradução das citações em língua inglesa. "A literacia, portanto, requer um engajamento ativo e autônomo com publicações, e exige o papel do indivíduo na medida em que ele produza, além de receber, atribuindo às mensagens interpretações independentes. A partir das competências básicas pressupostas para a literacia, as literacias de informática, cultural, econômica e outras, evoluíram como atributos de uma competência mínima exigida nessas áreas.” (HARRIS \& HODGES, 1995, p. 19).
} 
propomo-nos explorar a diversidade de perspetivas em que são utilizados nos artigos científicos circunscritos às bases de dados ISI (ISI Web of Knowledge) e ERIC (Education Resources Information Center).

Em primeiro lugar, a expressão literacia digital, embora seja frequente, tanto em contextos académicos como no dia do cidadão, encerra significados diversos e nem sempre convergentes. O interesse da comunidade científica pela temática tem vindo a aumentar na última década. Comprovam-no o número de estudos publicados na base de dados ISI Web of Knowledge, como podemos ver no gráfico abaixo.

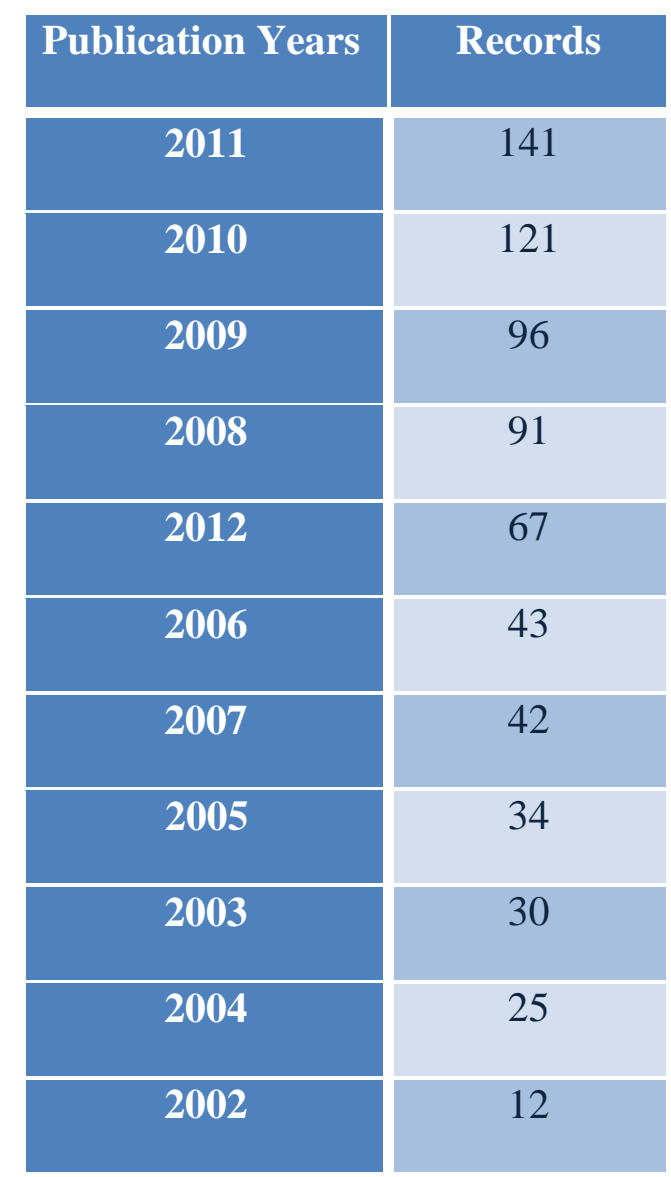

Gráfico 1 - Evolução do número de artigos publicados na base de dados ISI Web of Knowledge na última década sobre o tema digital literacy.

O gráfico exportado diretamente da ISI Web of Knowledge, da base de dados Current Contents Connect, revela claramente o interesse crescente por parte da comunidade científica em aprofundar a investigação em torno da expressão digital literacy. Desde o ano de 2002 que têm crescido o número de estudos. Salvaguardamos os dados provisórios relativos a 2012, pois representam praticamente os resultado de meio ano de atividade. 


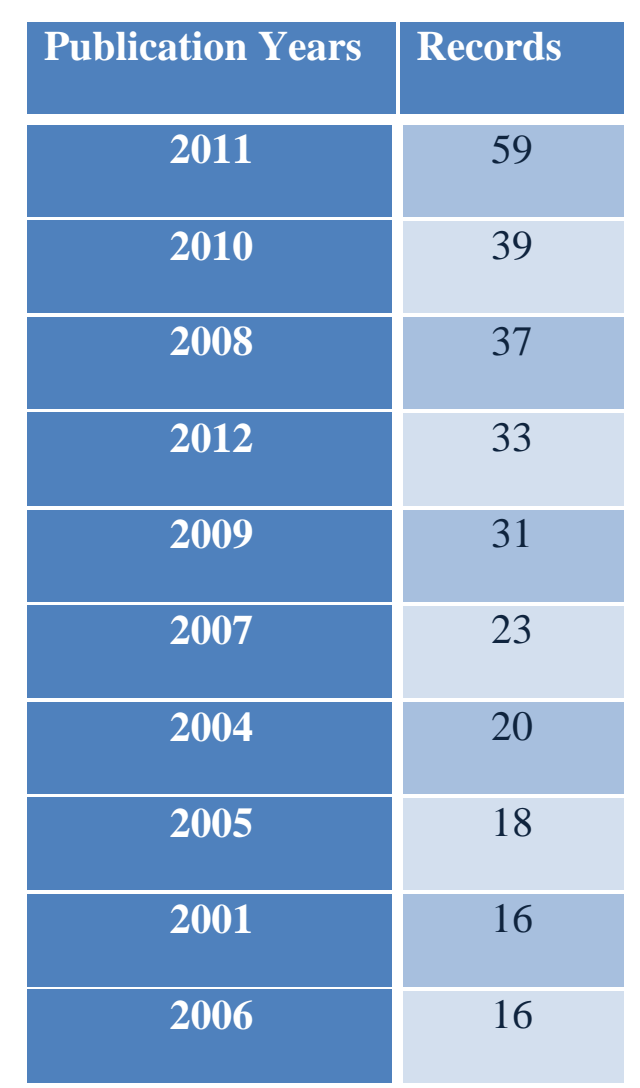

Gráfico 2 - Evolução do número de artigos publicados na base de dados ISI Web of Knowledge na última década sobre o tema informational literacy.

Interesse semelhante tem ocorrido em torno da expressão informational literacy, por parte da comunidade científica. Embora sem a expressão dos números anteriores, podemos constatar que entre 2000 a 2007 a variação de estudos não se poderá considerar estatisticamente relevante. Por outro lado, a partir do 2008 o número de investigações tende a estabilizar para depois, a partir do ano 2010 crescer de forma exponencial, como comprova o ano de 2011 com 59 resultados. Uma nota final para o ano de 2012, atendendo que os 33 resultados mostram somente a tendência do primeiro semestre.

Face, então, à amplitude semântica das expressões digital literacy e informational literacy percepcionada genericamente e à plurissignificação do seu uso, é nossa intenção conhecer os conceitos a elas associados, unicamente em artigos científicos, bem como explorar a implicação desta problemática na educação. Propomo-nos, então, fazer uma revisão sistemática da literatura, cujo processo é determinado a partir de um protocolo cuja delimitação processual será devidamente descrito mais à frente. 


\section{Q̨uadro teórico}

Qualquer que seja a área de investigação, a revisão de literatura assume uma posição determinante na prossecução dos objetivos definidos pelo investigador e pelo legado que irá facultar à comunidade científica. Caso não seja bem orientada, poderá mesmo enviesar os pressupostos do estudo ou deturpar os resultados.

É por demais evidenciado que os avanços tecnológicos mudaram de tal forma o mundo que, segundo Friedman, (2005) autor de The World is Flat: A Brief History of the Twenty-First Century, o transformaram de redondo em plano.

Apropriamo-nos da poderosa metáfora de Friedman para refletir sobre os referenciais que sustentam, como âncoras, as publicações a que se recorre para fundamentar teorias, correntes de pensamento ou mesmo opções por determinados campos de ação. Porém, na hora de construir esse corpus referencial que pode levar a compreender a aceção dos termos digital lteracy ou informational literacy, emergem cada vez mais dúvidas na definição de critérios explícitos na pesquisa de artigos e autores que configurem um constructo processual apoiado em metodologias científicas.

O assunto não é novo. Na altura em que surgiram as primeiras universidades na Europa, Tomás de Aquino decidiu por ordem no saber e construiu uma vigorosa síntese filosófica-teológica a partir do pensamento aristotélico e escreveu a Summae Theologiae. O filósofo medieval levou por diante essa empresa que perdura até aos nossos dias. Já no nosso tempo, Harold Bloom escreveu o Cânone Ocidental. Nesta obra, alimentada presumivelmente pela angústia de fazer a melhor escolha na hora de selecionar a melhor leitura, Bloom arrisca estabelecer uma lista de obras que supostamente formam o cânone ocidental ou, traduzido numa expressão mais informal, propõe o melhor dos melhores.

Neste estudo exploratório vamos, justamente, tentar compreender a amplitude das expressões, no atual contexto marcado por profundas transformações técnicas e tecnológicas a que assistimos e participamos. De forma muito particular, no panorama editorial, em poucos anos deparamo-nos perante um espetro de publicações e de divulgação bibliográfica sem precedentes (HART, 2006). E daqui surge uma questão fundamental - que procedimentos se devem, então, ter presentes numa revisão de literatura?

Habitualmente, em projetos de investigação, depois da definição do tema a estudar, e para conhecer o estado da arte relativamente ao tópico, estuda-se a bibliografia disponível, ou seja, os autores e obras considerados relevantes pela comunidade científica da área em estudo. Procura-se deste modo obter uma síntese do estado do conhecimento relativo à problemática 
em estudo. Em palavras de Creswell, o investigador deve começar "com uma revisão de literatura [o que] o ajuda a limitar o escopo da sua investigação e transmite para os leitores a importância de estudar um tópico” (2007, p. 43). O mesmo autor reconhece não haver "um modo único de conduzir a revisão de literatura” e sugere alguns passos para esse processo: "passo 1- comece identificando as palavras-chave úteis para localizar materiais na biblioteca de uma faculdade; passo 2 - com essas palavras-chave em mente, vá à biblioteca e comece a pesquisar o catálogo em busca de títulos (por exemplo periódicos e livros) [...] e pesquisar em bancos de dados online como ERIC, PsychoINFO” (2007, p.49).

Creswell recomenda que os investigadores estabeleçam uma prioridade na pesquisa bibliográfica: “comece com visões gerais encontradas em enciclopédias (...) artigos de periódicos respeitados (...) procure livros relacionados ao tópico, monografias, livros completos, (...) trabalhos de conferências recentes (...) artigos em web sites também são materiais úteis” (pp. 53-54).

As palavras de Creswell, que em outros tópicos relativos à investigação em educação muito respeitamos, ilustram as estratégias generalistas, frequentemente seguidas para recolher bibliografia para a Revisão de Literatura. Também nós o fizemos até há dois anos atrás.

Cremos, todavia, que o desafio passará por re-construir um caminho conceptual e metodológico adequado aos objetivos de cada investigador, num tempo em que a produção científica é diferente do passado. Precisamos, então, de atribuir ou reconhecer validade científica nas obras que constituem esse corpus, seguir procedimentos para que os resultados não sejam incompletos, ineficientes ou, em última análise, sem validade científica conforme defendem investigadores ligados às Ciências Socais (I. SAUR-AMARAL, 2011; TRANFIELD, DENYER, \& SMART, 2003).

Pelos dados que dispomos, verificamos que em Portugal, como em outros países, não são conhecidos ainda grandes avanços na área da Revisão Sistemática de Literatura das Ciências da Educação. No entanto, têm-se intensificado as publicações neste âmbito como se pode consttatar pelos autores referenciados (GOUGH, TRIPNEY, KENNY, \& BUK-BERGE, 2011; LEVIN, COOPER, ARJOMAND, \& THOMPSON, 2011; REES \& OLIVER, 2012; SQUIRES \& ET AL., 2011; STEWART \& OLIVER, 2012; VANDERLINDE \& VAN BRAAK, 2010). Noutras áreas da investigação, a Revisão Sistemática tem revelado influência profunda nos resultados dos estudos onde é aplicada, nomeadamente nas Ciências Médicas (CONTANDRIOPOULOS, LEMIRE, DENIS, \& TREMBLAY, 2010).

Merece, neste contexto, uma consideração especial dois exemplos que ilustram bem a importância de compreender este movimento concetual de disciplinar de certa forma a revisão 
de literatura: o projeto em curso Evidence Informed Policy and Practice in Education in Europe (2011-13) inscrito nos programas da Comunidade Europeia e que visa aumentar a utilização de provas para informar a tomada de decisões na política educacional e prática em toda a Europa e, também, os trabalhos desenvolvidos pela associação Campbell Collaboration, sediada na Noruega, cujo principal intuito é ajudar investigadores e outros profissionais a tomar decisões bem informadas a partir de revisões sistemáticas. Os princípios que regem estas duas iniciativas são comuns e similares nos pressupostos epistemológicos. Essa ideia comum está muito bem sintetizada no portal da Campbell Collaboration, quando afirma: "the purpose of a systematic review is to sum up the best available research on a specific question. This is done by synthesizing the results of several studies. A systematic review uses transparent procedures to find, evaluate and synthesize the results of relevant research" ${ }^{2}$.

Subjacente aos exemplos apresentados está um entendimento de revisão de literatura enquanto processo que deverá passar por etapas devidamente delineadas e definidas em todos os momentos, desde os objetivos a que se propõe até aos procedimentos metodológicos, como defendem vários investigadores ligados às Ciências Sociais (DENYER \& TRANFIELD, 2009; I. SAUR-AMARAL, 2010). Segundo estes autores, o processo de revisão da literatura, ao estar inscrito e descrito no desenho metodológico da investigação, deve esclarecer o modo como foram selecionadas e apuradas as fontes, de modo que as conclusões a extrair sobre os assuntos em estudo possam ser cientificamente consistentes.

A revisão sistemática caracteriza-se, por conseguinte, por empregar uma metodologia de pesquisa com rigor científico e de grande transparência, cujo objetivo visa minimizar o enviesamento da literatura, na medida em que é feita uma recolha exaustiva baseada em critérios objetivos dos textos publicados sobre o tema em questão (THORPE, HOLT, MACPHERSON, \& PITTAWAY, 2005; TRANFIELD, DENYER, \& SMART, 2003). Assim, é basilar o objetivo de estruturar todos os procedimentos de forma a garantir a qualidade das fontes, desde logo pela definição de uma equação de pesquisa, de critérios de inclusão e exclusão e de todos os critérios que julguem convenientes para o caso. A credibilidade da pesquisa será, desta forma, proporcional ao cuidado de como se estabelecem as regras, pela possibilidade de replicação do processo por um outro indivíduo, na medida em que aquilo que não é verificável pelos pares, não é científico (HOLLIS, 1994; HUGHES,

\footnotetext{
2 <http://www.campbellcollaboration.org/what_is_a_systematic_review/index.php>. “a finalidade de uma revisão sistemática é fazer um resumo das melhores pesquisas disponíveis a respeito de uma questão específica. Isto é feito sintetizando os resultados de diversos estudos. Uma revisão sistemática utiliza procedimentos determinados com transparência para encontrar, avaliar e sintetizar os resultados de pesquisas relevantes”.
} 
1997).

Deveremos, assim, procurar definir critérios, métodos precisos e sistemáticos, por forma a identificar e selecionar as fontes bibliográficas com o máximo rigor, grau de eficiência e ponto de oferecer confiança no trabalho desenvolvido. O recurso ao software EndNote contribuirá para operacionalizar os processos a seguir descritos na gestão de referências e citações bibliográficas de que daremos parte ao longo do artigo.

\section{Metodologia de pesquisa}

O processo de Revisão Sistemática implica tematizar a questão e objetivar princípios que colaborem na definição de um pensamento conducente a uma experiência de seleção de fontes afastado o mais possível de convicções pessoais ou de avaliações subjetivas influenciadas por coordenadas geográficas ou pela pertença a uma determinada comunidade académica (BRINER \& DENYER, 2012).

O pensamento de David Gough e colaboradores (GOUGH, THOMAS, \& OLIVER, 2012; GOUGH, TRIPNEY, KENNY, \& BUK-BERGE, 2011), um dos investigadores mais proeminentes na área da educação, atualmente, e outros académicos (SLAVIN, 2008; WARD, HOUSE, \& HAMER, 2009) põem em confronto as duas formas distintas de levar por diante a tarefa de se realizar uma revisão de literatura, sublinhado neste excerto:

traditional literature reviews typically present research findings relating to atopic of interest. They summarise what is known on a topic. They tend to provide details on the studies that they consider without explaining the criteria used to identify and include those studies or why certain studies are described and discussed while others are not. Potentially relevant studies may not have been included because the review author was unaware of them or, being aware of them, decided for reasons unspecified not to include them. If the process of identifying and including studies is not explicit, it is not possible to assess the appropriateness of such decisions or whether they were applied in a consistent and rigorous manner ${ }^{3}$ (p.5).

Interessa aqui problematizar e apresentar uma perspetiva renovada do estado da arte a propósito de um assunto com interesse investigativo na área das Tecnologias de Informação e

\footnotetext{
3 “Revisões literárias tradicionais tipicamente apresentam descobertas de pesquisa relacionadas a um assunto de interesse. Resumem o que se sabe sobre um assunto. Tendem a fornecer detalhes sobre os estudos que são considerados sem explicar os critérios utilizados para identificar e incluir tais estudos, ou justificar por que certos estudos são descritos e discutidos, e outros não. Estudos potencialmente relevantes podem não ter sido incluídos porque o autor da revisão não tinha conhecimento deles, ou pode-se deixar de incluí-los por motivos não explicados. Se o processo de identificação e inclusão de estudos não é explícito, não é possível acessar o quão apropriadas são tais decisões, ou se elas foram aplicadas de maneira consistente e rigorosa.”
} 
Comunicação - o entendimento do conceito de literacia digital na base de dados ISI Web of Knowledge (ISI) e da Education Resources Information Center (ERIC) nos últimos cinco anos.

Apresentamos um modelo de pesquisa a partir de um protocolo definido para o efeito, do qual constam: (i) objetivos (ii) equações de pesquisa pela definição dos operadores booleanos; (iii) âmbito; (iv) critérios de inclusão; (v) critérios exclusão; (vi) critérios de validade metodológica; (vii) resultados; (viii) tratamento de dados.

De forma esquemática, poderia ser assim sintetizado:

\begin{tabular}{|c|}
\hline OBJETIVOS \\
\hline Definir a problemática a estudar sintetizada numa questão ou problema \\
\hline EQUAÇÕES DE PESQUISA \\
\hline Expressões ou palavras as combinar utilizando AND, OR, NOT (*, ?) \\
\hline ÂMBITO DA PESQUISA \\
\hline Bases de a selecionar e variantes intrínsecas \\
\hline CRITÉRIOS INCLUSÃO \\
\hline Definem que o estudo é aceitável naquele contexto \\
\hline CRITÉRIOS DE EXCLUSÃO \\
\hline Excluem os estudos que não obedecem ao âmbito definido \\
\hline Asseguram a objetividade da pesquisa \\
\hline Riltrar e analisar criticamente os resultados com apoio de software de gestão bibliográfica (EndNote) \\
\hline TRATAMENTO DOS DADOS \\
\hline Devem ser registados todos os passos \\
\hline
\end{tabular}

Gráfico 3 - Etapas do protocolo de Revisão Sistemática.

(Adapt. de Saur-Amaral (2011 ), Tranfield \& Mouchel (2002) e Gough, Thomas \& Oliver (2012).

Convém, entretanto, clarificar que esta série de procedimentos pode ser realizada com mais ou menos passos, conforme acontece noutros âmbitos do conhecimento perfeitamente familiarizados com esta conceção de revisão de literatura. Este procedimento é, na nossa perspetiva, o que nos parece mais equilibrado, exequível e aplicável no âmbito das investigações produzidas nas Ciências da Educação. Apesar de todos os passos desta proposta terem objetivos e finalidades definidas, o último ponto - tratamento de dados -, merece ser sublinhado pela sua relevância no decurso do processo. Filtrar, organizar, analisar criticamente os resultados das pesquisas em bases de dados online, como é o caso da ISI e da 
ERIC, só se torna procedente com o auxílio de um programa de gestão bibliográfica, como será um excelente exemplo o conceituado EndNote. Depois da seleção dos textos a incluir na revisão de literatura, este programa constituirá um excecional recurso para organizar referências bibliográficas, com facilidade de acesso e atualização de forma simples e devidamente sincronizadas com o processador de texto Word.

\section{Resultados da pesquisa}

Neste passo, detalhamos e concretizamos todo o processo realizado o qual se sintetiza no gráfico a seguir, com os resultados obtidos.

\begin{tabular}{|c|c|}
\hline $\begin{array}{l}\text { FASES DO } \\
\text { PROTOCOLO }\end{array}$ & DESCRIÇÃO DAS ETAPAS \\
\hline Objetivos & $\begin{array}{l}\text { Identificar artigos que tratem o conceito de literacia digital } \\
\text { associados à educação, para que se estabeleça as aceções } \\
\text { dominantes pelos vários autores. }\end{array}$ \\
\hline Equações de pesquisa & digital literacy; informational literacy \\
\hline Âmbito da Pesquisa & $\begin{array}{l}\text { ISI Web of Knowledge e Educattion resources Information } \\
\text { Center, nos últimos cinco anos (2008-12). }\end{array}$ \\
\hline Critérios de inclusão & Só serão aceites artigos publicados em revistas científicas. \\
\hline Critérios de exclusão & $\begin{array}{l}\text { Artigos sem sumário, artigos publicados noutras fontes e fora } \\
\text { do âmbito educacional que não sejam em inglês e português. }\end{array}$ \\
\hline $\begin{array}{l}\text { Critérios de validade } \\
\text { metodológica }\end{array}$ & $\begin{array}{l}\text { Replicação do processo por dois investigadores; Verificação dos } \\
\text { critérios de inclusão e exclusão; }\end{array}$ \\
\hline Resultados & Descrição da pesquisa - Registo de todos os passos. \\
\hline Tratamento de dados & $\begin{array}{l}\text { Filtrar, analisar e descrever criticamente os resultados com } \\
\text { auxílio do EndNote e do NVivo. }\end{array}$ \\
\hline
\end{tabular}

\section{Gráfico 4 - Descrição das etapas seguidas no processo de Revisão Sistemática}

Seguindo o esquema, a pesquisa foi desenvolvida no dia 4 de julho de 2012, tendo como intuito compreender a conceção dos termos digital literacy e informational literacy, em artigos científicos associados à educação nas bases de dados ISI (Current Contents Connect) e ERIC. Após definido o âmbito temático, interessava agora mapear nas bases de dados referenciais a ocorrência do termo, seguindo determinados critérios.

Face à especificidade funcional intrínseca a cada uma das bases de dados, optamos por 
ajustar os meios de busca sem alterar o sentido e o âmbito dos pressupostos definidos. Quer numa situação, quer noutra, optou-se por iniciar todo o processo em "busca avançada" de forma a podermos circunscrever o melhor possível o objeto em estudo. Em primeiro lugar, comum à ISI e à ERIC, só foram aceites artigos com peer reviewed, como frequentemente se designa por artigos com arbitragem científica. Para além deste critério de inclusão, também só consideramos no acervo os artigos escritos em língua inglesa e publicados a partir do ano 2008 até 2012. De salientar ainda que por se tratar de um estudo exploratório, os dados em estudo limitam-se aos resumos, estratégia sugerida pela Revisão sistemática de Literatura para uma abordagem inicial à temática em estudo. Atendendo à particularidade da base referencial ERIC permitir, à partida, a definição do tipo de documento, decidimos por apresentar individualmente os procedimentos de pesquisa. No caso da pesquisa realizada na base de dados bibliográfica ERIC, construiu-se a seguinte equação de pesquisa: selecionamos o campo advanced search a partir da expressão digital literacy, alargámos a pesquisa a keywords, sem utilizar qualquer operador booleano; depois, foi acionado a função peer review; o intervalo de pesquisa foi estabelecido entre os anos 2008 a 2012; quanto ao tipo de documento, selecionamos somente jornal articles. Com a definição desta equação de pesquisa, o resultado parcial foi de 72 ocorrências.

A seguir, o processo de pesquisa foi replicado na base de dados multidisciplinar ISI Web of Knowledge. Porém, atendendo às suas características de configuração, começamos por limitar a área education educational research, aberta ao campo topic e com o mesmo termo de pesquisa. O resultado foi de 49 ocorrências.

Depois de exportados todos os resumos dos artigos de ambas as bases de dados, no total de 121 resultados com potencial interesse, no EndNote procedeu-se ao refinamento dos resultados. Foram encontrados 13 artigos duplicados, portanto comuns às duas bases de dados, tendo o número sido reduzido para 108. Exportados os resumos para o processador de texto, todo o trabalho de análise foi realizado a partir daqui. Neste caso, o trabalho de seleção exigiu um grau de filtragem ainda maior, porque alguns deles não se enquadravam no âmbito dos pressupostos iniciais. O total dos artigos que serviram de base à redação final é constituído por 90 .

Na pesquisa realizada na base de dados bibliográfica ERIC, todo o processo foi replicado para a expressão informational literacy. Detalhamos em seguida a equação de pesquisa: selecionado o campo advanced search, a partir da expressão informational literacy, alargámos a pesquisa a keywords, sem utilizar qualquer operador booleano; depois, foi acionada a função peer review; o intervalo de pesquisa foi estabelecido entre os anos 2008 a 
2012; quanto ao tipo de documento, selecionamos somente jornal articles. Com a definição desta equação de pesquisa, o resultado parcial foi de 45 ocorrências.

Seguidamente, o processo de pesquisa foi replicado na base de dados multidisciplinar ISI Web of Knowledge. Atendendo às suas características de configuração, começamos por limitar a área education educational research, restrita agora ao campo title, com o mesmo termo de pesquisa de forma a focalizar a busca. O resultado obtido foi de 22 ocorrências.

Depois de exportados todos os resumos dos artigos de ambas as bases de dados, no total de 67 resultados com potencial interesse; no EndNote procedeu-se ao refinamento dos resultados. Foram encontrados quatro artigos duplicados, portanto comuns às duas bases de dados, tendo o número sido reduzido para 63. Exportados os resumos para o processador de texto, todo o trabalho de análise foi realizado a partir daqui. Neste caso, o trabalho de seleção exigiu um grau de filtragem ainda maior, porque alguns deles não se enquadravam no âmbito dos pressupostos iniciais. O total dos artigos que serviram de base à redação final é constituído por 63 artigos.

Perspetiva analítica dos artigos apurados:

\begin{tabular}{|l|c|c|c|}
\hline \multicolumn{3}{|c|}{ Mapa } \\
\hline $\begin{array}{c}\text { ERIC (Education } \\
\text { Resources } \\
\text { Information Center) }\end{array}$ & $\begin{array}{c}\text { ISI Web of } \\
\text { Knowledge (Current } \\
\text { Contents Connect) }\end{array}$ & $\begin{array}{c}\text { Total de artigos } \\
\text { apurados }\end{array}$ \\
\hline Digital literacy & 72 & 49 & 90 \\
\hline Informational & 45 & 22 & 63 \\
\hline literacy & 111 & 71 & 182 \\
\hline TOTAL & & & \\
\hline
\end{tabular}

Gráfico 5 - Mapa geral dos artigos apurados na Revisão Sistemática

\section{Metodologia de análise}

$\mathrm{Na}$ investigação qualitativa os dados são frequentemente volumosos e não estruturados, o que pode tornar a sua análise morosa e complexa. Por outro lado, é necessário ter fácil acesso ao contexto em que ocorrem determinados fenómenos, uma vez que o sentido é quase sempre dependente desse contexto. Estas características, e ainda o facto de a investigação qualitativa ser um processo interativo, sujeito a constantes reformulações, 
dificultam a gestão, o tratamento e a análise dos dados. O computador, pela facilidade e flexibilidade que permite na organização e reorganização dos dados, encoraja o investigador a experimentar diferentes perspetivas de abordagem, o que é difícil, ou mesmo impossível, com recursos tradicionais.

Atualmente, existe uma grande variedade de software de apoio à análise qualitativa. Optamos pelo programa informático NVivo10 por ser uma ferramenta reconhecida na comunidade científica, permitir analisar texto, som, imagem fixa, vídeo e, sem ser necessária a transcrição, ainda que esta tenha, a nosso ver, várias vantagens. É também possível importar, analisar e exportar dados quantitativos, sociodemográficos e outros. Este software permite ainda recolher diretamente dados da internet - Facebook, Twitter, Wordpress, por exemplo, o que o torna cada vez mais poderoso para muitas das investigações em curso na área da utilização educativas das TIC. No caso deste estudo, apenas foram usados dados textuais.

O NVivo adapta-se a diferentes desenhos metodológicos, já que é a metodologia que orienta o uso que o investigar faz do software e não o contrário. Com efeito, apoia a interpretação e a análise do investigador sendo suficientemente flexível para acompanhar todas as reformulações que frequentemente ocorrem durante o processo. O conjunto de ferramentas de questionamento dos dados (texto ou relações entre categorias) do NVivo, estimula o investigador a explorar padrões e testar hipóteses facilitando a dimensão mecânica desse trabalho.

Neste artigo utilizamos o NVivo para conhecer, clarificar e delimitar os conceitos de digital literacy e informational literacy. De modo a facilitar a apresentação de resultados, apresentamos e comentamos um modelo exploratório produto da análise dos dados. Trata-se, no entanto, de uma abordagem inicial e exploratória a esta temática que continuará a ser objeto de estudo da nossa parte.

\section{ANÁLISE E APRESENTAÇÃO DE RESULTADOS}

No que diz respeito a digital literacy, dos 90 resumos analisados, sete foram do ano de 2008; 21 de 2009; 22 de 2010; 32 de 2011 e oito de 2012. Este baixo número em 2012 devese, com certeza, ao facto de a recolha ter sido efetuada no dia 4 de Julho 2012. Quanto aos 63 resultados apurados na pesquisa de informational literacy, foram 10 relativos ao ano de 2008; nove de 2009; 20 de 2010; 16 de 2011; seis de 2012. Foram ainda consultados dois dicionários: o da Academia das Ciências de Lisboa (2001) e o The literacy dictionary. The 
vocabular of reading and writing, editado por Harris e Hodges (1995).

Depois de uma leitura extensiva e intensiva dos dados obtidos, os textos foram importados para a base de dados do NVivo como fontes internas. Antes da categorização do corpus, fez-se a tabela de frequências (palavras gramaticais omitidas) e posterior nuvem das palavras mais frequentes do texto.

access activities among analysis approach article available based basic children classroom collaborative communication community computer contains content contexts course critical current design develop developed development different

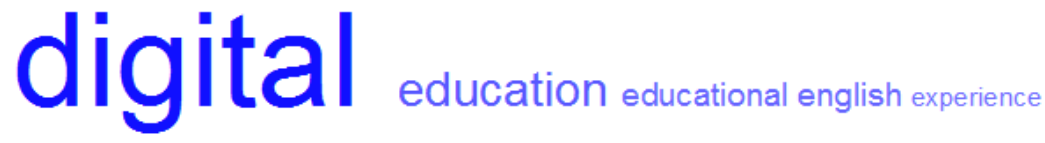
findings focus games group groups however impact information inquiry

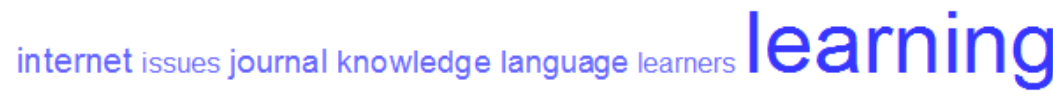
neaseses literacy paper participants people policy practice practices production professional program project public purpose questions related reports $\mathrm{reSe}$ a $\mathrm{rCh}$ results school schools science sites Skills social society sources student students students' studies study support survey teacher teachers teaching technologies technology texts three tools training

Figura 1 - Mapa de frequências das palavras para Digital Literacy (Tag Clouds) dos resumos.

Em relação a digital literacy verifica-se que as ocorrências mais frequentes são, por ordem decrescente: literacy (248); digital (233); learning (143); student (91); information (79); research (75), o que anuncia desde logo as problemáticas mais relevantes no corpus estudado. 


\title{
12200820092010201134 access article assessment attention based c childhood competence computer computers contains course critical current curriculum data development digital early education educational elsevier evidence feedback finding findings first focus framework group health high higher il
}

\section{information}

informational instruction

\section{internet k knowledge lack learning libraries library $I$ iter}

one online paid paper parents perception problem problems project public purpose

\section{research results school schools search searching significant skill}

\author{
skills solving standards strategies structured student $\mathbf{S t u d e n t}$ students' \\ studies Study support survey table teachers teachers' teaching technology \\ thinking three training two Use using video well year
}

Figura 2 - Mapa de frequências das palavras para Informational Literacy (Tag Clouds) dos resumos.

Quanto a digital informational as ocorrências mais frequentes são, por ordem decrescente: information (134); literacy (79); research (54); students (43); skills (34); education (27), o que anuncia desde logo as problemáticas mais relevantes no corpus estudado.

Em comum, embora com frequências absolutas diferentes, encontramos literacy, information e research o que decorre não tanto do número de artigos encontrado para cada um dos conceitos mas mais, a nosso ver, do enfoque dos respetivos artigos como adiante referimos.

A figura seguinte apresenta as categorias que sintetizam os resultados da análise e que se referem a: 1) dimensões específicas de cada um dos conceitos em estudo, tendo sido selecionadas cinco que consideramos mais relevantes em cada um e as inter-relações entre algumas delas, no caso da Literacia Informacional; 2) dimensões comuns aos dois conceitos, duas; 3) contextos em que, segundo os autores, devem ser desenvolvidas, apenas no caso da Literacia Informacional; e 4) justificação da absoluta necessidade de desenvolver desde muito cedo tanto a Literacia Digital como a Literacia Informacional.

O texto descritivo e interpretativo é ilustrado com excertos da evidência recolhida nos resumos com base na qual se procedeu à categorização em NVivo. 


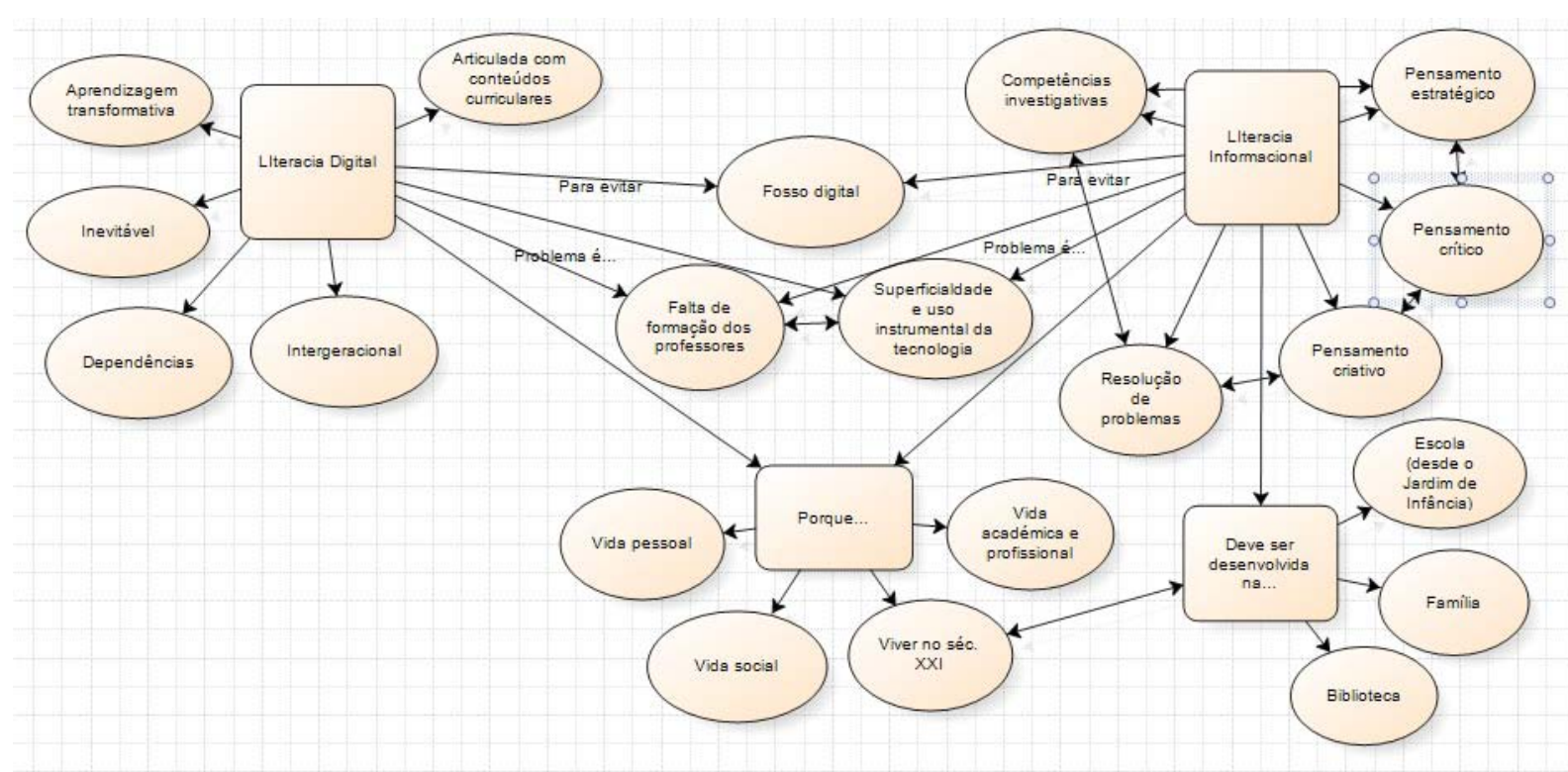

Figura 3 - Modelo exploratório relativo aos conceitos de Literacia Digital e Literacia Informacional

\section{Dimensões da Literacia Digital}

- Aprendizagem transformativa (“digital literacies are transformative for pedagogy"4): vários são os autores que consideram que só existe literacia digital quando há uma verdadeira transformação das práticas do sujeito tanto na sua vida profissional como pessoal;

- Problemática intergeracional ('Through an eighty-one-year-old woman's literacy narrative, I argue that literacy researchers should pay greater attention to elder writers”5): embora não seja uma temática frequente, alguns dos resumos salientam que a literacia digital deve abranger os adultos e mesmo os idosos como forma de contribuir para o seu desenvolvimento pessoal e integração social;

- Inevitabilidade da literacia digital ("the most solid of ground to be found in the debate surrounding digital literacy is the agreement that, whatever it is, it is important to the success of our students"6): com efeito, hoje já não se discute se sim ou não o cidadão,

\footnotetext{
4 “Literacias digitais são transformadoras para a pedagogia”.

5 "Por meio da literacia narrada por uma mulher de oitenta anos de idade, argumento que pesquisadores da literacia deveriam dedicar atenção a escritores mais velhos.”

6 “O alicerce mais sólido no debate acerca da literacia digital é o acordo de que, o que quer que seja, ela é
} 
particularmente crianças e jovens, precisam de usar tecnologia, elas são indispensáveis pelo menos para o seu sucesso académico e profissional.

- Dependências que podem surgir decorrentes do desenvolvimento da literacia digital. Em relação às possíveis desvantagens ou limitações do uso das tecnologias, as que surgem nos resumos estudados são comuns na literatura específica da área. As características consideradas negativas distribuem-se por vários domínios e prendem-se com: aspetos emocionais, como a ansiedade decorrente da quantidade de informação que nos inunda cada dia o que pode gerar insegurança ao não se ser capaz de dominar toda a informação; aspetos cognitivos como a sobrecarga decorrente da acumulação de tarefas que as tecnologias acabam por desencadear. Há ainda o que se prende com as características de muita da informação que circula online: a fragmentação, a superficialidade, o imediatismo, e por vezes a incorreção, o que requer do utilizador competências de pesquisa, análise e seleção crítica a que, no contexto deste estudo, uns autores designam por literacia digital, outros informacional como adiante referimos.

Articulação da literacia digital com conteúdos curriculares ("the wiki experience... engaged students in interactional communication in the second language, encouraged negotiation of meaning, and challenged learners in finding their "solutions" to real life problems around them, aside from acquisition of hands-on digital literacy"77): alguns consideram que a literacia digital não deve ser trabalhada como um conteúdo independente, antes deve ser integrada no tratamento de outros conteúdos. O facto de a tecnologia ser contextualizada na aprendizagem de conteúdos curriculares permite ao aluno ver a sua dimensão cognitiva além da meramente instrumental.

\section{Dimensões da Literacia Informacional}

Enquanto nos resumos relativos à Literacia Digital foi possível discriminar dimensões autónomas ainda que complementares, relativamente à Literacia Informacional o mesmo não acontece na grande maioria dos resumos. Com efeito, e como as setas bidirecionais no modelo acima evidenciam, as cinco dimensões Pensamento crítico, Pensamento criativo, Pensamento estratégico, Competências investigativas e Resolução de problemas surgem quase sempre

\footnotetext{
importante para o sucesso dos nossos estudantes”.

7 “A experiência wiki... estudantes engajados em comunicações interativas nessa linguagem que incentiva a negociação de significados, e desafia os alunos a encontrar soluções para problemas da vida real ao seu redor, além de adquirirem literacia digital na prática.”
} 
associadas, tenham estas ou outras denominações conceptualmente próximas. Este facto remete-nos, desde logo, para as competências de pensamento de ordem superior subjacentes à Literacia Informacional. Eis a evidência que o comprova: "[s]tudents who are information literate develop strategic, critical, and creative thinking dispositions that serve both their personal and educational needs"; "students [need] to be creative and dogged in their research, to think critically about the information they find, and to provide solutions and tools for fellow students for improving their research and critical thinking skills"; "[f]ew issues in higher education are as fundamental as the ability to search for, evaluate, and synthesize information. The need to develop information literacy, the process of finding, retrieving, organizing, and evaluating the ever-expanding collection of online information"; "[r]esearch suggests that early information literacy instruction (...) promotes critical thinking and increases the ability to problem-solve: two skills necessary for survival in today's Information Age" $^{8}$.

\section{Dimensões comuns à da Literacia Digital e à Literacia Informacional}

Da análise dos resumos dos artigos relativos aos dois conceitos em estudo emergem três categorias comuns: Fosso digital, Superficialidade e uso instrumental das TIC e Formação de Professores a nosso ver por serem tópicos transversais tanto a este tema específico como à problemática geral do uso educativo de recursos digitais.

- Fosso digital ("[c]urrent European initiatives focus on providing access to a PC with internet and ensuring basic usage skills to address the digital divide"; "reduction of the digital divide between students [are critical for their success] ${ }^{9 "}$ : é indispensável que a educação formal contribua para atenuar as diferenças sociais que ainda existem entre os

\footnotetext{
8 "estudantes que possuem literacia informacional desenvolvem dispositivos para o pensamento estratégico, crítico e criativo, que servem tanto às suas necessidades pessoais quanto às educacionais"; "os estudantes [precisam] ser criativos e estar mergulhados nas suas pesquisas, pensar criticamente sobre as informações que encontram, providenciar soluções e ferramentas para seus colegas para o aprimoramento das suas pesquisas e suas habilidades de pensamento crítico"; "poucas questões de educação superior são tão fundamentais quanto a habilidade de buscar, avaliar e sintetizar informações. A necessidade de desenvolver literacia informacional, o processo de encontrar, recolher, organizar e avaliar a coleção de informações virtuais que está em constante expansão"; "pesquisa sugere que a instrução para a literacia informacional desde cedo (...) promove o pensamento crítico e aumenta a habilidade em resolver problemas: duas ferramentas que são necessárias para a sobrevivência na atual Era da Informação.”

9 "Recentes iniciativas europeias dedicam-se a promover acesso a PCs com internet a garantir conhecimentos básicos ao abordar a exclusão digital"; "redução da exclusão digital entre estudantes [é essencial para seu sucesso]"
} 
indivíduos. Em Portugal, a iniciativa Plano Tecnológico para a Educação foi um importante contributo nesse sentido.

- Superficialidade e uso instrumental das TIC ("the majority of students performed basic office duties such as greeting faculty, logging computers, as well as answering phones”; "students need to hone their research skills, moving beyond a dependence on Google and Wikipedia"; " [a]nalysis of the survey revealed a significantly higher percentage of students who provided incorrect responses reported using Google as their preferred online search method"; "[students] lack the critical thinking skills required to successfully evaluate the actual credibility of online information, a critical aspect of information literacy"10): ambos os conjuntos de resumos referem este tipo de utilização quase só instrumental e sem qualquer impacto, nem na aprendizagem, nem no desenvolvimento intelectual dos estudantes.

- Formação de Professores ("evidence also revealed a range of concerns about lack of skills and knowledge needed to search and evaluate information effectively"; "[t]here have been many recent UK initiatives aimed at improving access to research evidence, but there are still concerns about the lack of engagement by teachers" ${ }^{11}$ ). Dos textos analisados emergem duas situações: é necessário, mas não é suficiente, saber usar tecnologias de modo instrumental; para muitos professores essa ainda é a utilização mais frequente não tendo desenvolvido as competências indispensáveis à pesquisa, tratamento e análise de dados que permite transformar a informação em conhecimento; nesse contexto, continua a ser necessário investir na formação dos professores para esse fim porque ela é determinante enquanto fator determinante no processo de aquisição e desenvolvimento das competências das literacias digital e informacional dos estudantes.

Remetemos para o ponto seguinte, Considerações Finais, a referência breve a duas categorias apresentadas no modelo exploratório, Porque e Desenvolvida em..., que não se relacionando diretamente com os conceitos de Literacia Digital e Literacia Informacional,

\footnotetext{
10 “A maioria dos estudantes executou tarefas básicas de trabalho como cumprimentar colegas e acessar computadores, bem como atender telefones”; "os estudantes precisam afiar suas ferramentas de pesquisa, indo além da dependência do Google e da Wikipedia”; “a análise do exame revelou uma percentagem significativamente maior de estudantes que apresentam respostas incorretas, afirmando se utilizar do Google como o método de pesquisa virtual de sua preferência”; “[estudantes] não apresentam as habilidades de pensamento critico necessárias para avaliar a real credibilidade das informações oferecidas na internet, um aspecto crucial da literacia informacional.”

11 "As evidências também revelaram uma série de preocupações quanto à falta de habilidades e conhecimento necessários para buscar e avaliar a efetividade das informações”; “foram feitas várias iniciativas do Reino Unido visando a melhoria do acesso a evidências de pesquisa, mas ainda há preocupações quanto à falta de envolvimento dos professores”
} 
tema deste artigo, permitem complementá-los e situá-los em contexto educativo.

\section{Considerações finais}

Ao iniciar esta análise julgávamos conseguir concretizar o que inicialmente prometíamos - conhecer, clarificar e delimitar os conceitos de literacia digital e literacia informacional - a partir de resumos. Concluímos, porém, que só o estudo dos artigos integrais correspondentes aos resumos analisados no-lo poderá permitir. Sabíamos, pela experiência de leituras anteriores, que não haveria consenso entre os autores. Também não existe entre os que agora estudamos: os dados analisados revelam que diferentes autores utilizam terminologias diferentes para se referirem a cada um dos conceitos.

No entanto, e salvaguardando, de novo, que se tratou de um estudo exploratório baseado apenas em resumos, técnica validada pela estratégia de Revisão Sistemática de Literatura, podemos afirmar que literacia digital aponta para usos elementares e instrumentais de recurso digitais e literacia informacional para uma utilização reflexiva e crítica, baseada em processos de pensamento de ordem superior, desses recursos, ao serviço da pesquisa, tratamento e análise da informação.

Os textos relativos ao conceito de literacia informacional enfatizam a urgência de desenvolver essa competência desde muito cedo e em diferentes contextos desde os ambientes formais aos não formais e informais, como bibliotecas e família, chamando a atenção para "the importance of fostering information literacy in home-school collaboration ${ }^{12}$ " e para a necessidade de "spend more class and homework time on informational literacy, and advocate for a more balanced approach to early childhood preparation across the content areas" ${ }^{13}$. De facto, alguns artigos argumentam que desde o Jardim de Infância os alunos devem ser habituados a não consumir passivamente a informação que encontram, donde "the importance of information literacy instruction in early childhood education", pois "research suggests that early information literacy instruction (...) promotes critical thinking and increases the ability to problem-solve, two skills necessary for survival in today's Information Age"14.

É já um lugar-comum dizer que, atualmente, o uso crítico e inteligente das tecnologias

\footnotetext{
12 "a importância do amparo à literacia informacional através de colaborações entre escola e casa”

13 "dedicar mais tempo em sala e em deveres de casa que lidem com a literacia informacional, e defendam uma abordagem mais balanceada do preparo na educação infantil desde cedo que abranja as áreas de conteúdo.”

14 “a importância da instrução para uma literacia informacional desde o início da educação infantil”, pois "pesquisas sugerem que uma precoce instrução para literacia informacional (...) promove o pensamento crítico e eleva a habilidade de resolução de problemas, duas ferramentas necessárias para a sobrevivência na atual Era da Informação."
} 
é indispensável, senão mesmo vital, para a vida académica, profissional, pessoal e social do indivíduo. Mas, e para lá do senso comum, o facto de esta ideia surgir com muita frequência e devidamente fundamentada nos artigos académicos nos quais baseamos esta análise, justifica em absoluto que essa seja uma prioridade no nosso trabalho de professores e educadores. Com efeito, e independentemente do debate académico acerca dos conceitos de literacia digital e literacia informacional, importa reter que "o rápido aumento da informação nos últimos 30 ou 40 anos torna impossível aos professores preparar os estudantes para o futuro sem os ensinar como ser gestores críticos e eficazes dessa informação" ${ }^{15}$.

\section{Referências}

ACADEMIA, D. E. C. D. E. L. Dicionário da Língua Portuguesa Contemporânea.Lisboa: Academia de Ciências de Lisboa e Editorial Verbo, 2001.

BRINER, R. B., \& DENYER, D. Systematic review and evidence synthesis as a practice and scholarship tool. Handbook of Evidence-Based Management: Companies, Classrooms and Research (This volume), . 2012

CONTANDRIOPOULOS, D., LEMIRE, M., DENIS, J. L., \& TREMBLAY, É. Knowledge exchange processes in organisations and policy arenas: a narrative systematic review of the literature. Millbank Quarterly, 88(4), 2010 (pp. 444-483).

DENYER, D., \& TRANFIELD, D. Producing a systematic review. (2009).

FRIEDMAN, T. L. The world is flat: a brief history of the twenty-first century (Vol. 5), 2005.

GOUGH, D., Thomas, J., \& Oliver, S.. Clarifying differences between review designs and methods. Systematic Reviews, 1(1), 28. 2012.

GOUGH, D., TRIPNEY, J., KENNY, C., \& BUK-BERGE, E. Evidence Informed Policy in Education in Europe: EIPEE final project report. London: EPPI-Centre, Social Science Research Unit, Institute of Education, University of London, 2011.

HARRIS, T. L., \& HODGES, R. E. The literacy dictionary: The vocabulary of reading and writing: International Reading Assn, 1995.

HART, C.. Doing a literature review: Releasing the social science research imagination: Sage, 2006.

HOLLIS, M. The philosophy of social science: an introduction. Cambridge: University Press, 1994.

HUGHES, J. A. The philosophy of social research / John A. Hughes, Wesley W. Sharrock. London; New York : Longman, 1997.

\footnotetext{
${ }^{15}$ Tradução dos autores.
} 
LEVIN, B., COOPER, A., ARJOMAND, S., \& THOMPSON, K. Research use and its impact in secondary schools. In E. Canadian Education Association Ontario Institute for Studies in (Ed.). Toronto: University of Toronto, 2011.

REES, R., \& OLIVER, S.. Stakeholder perspectives and participation in reviews. In D.

Gough, S. Oliver \& J. Thomas (Eds.), An Introduction to Systematic Reviews. London: Sage, 2012.

SAUR-AMARAL, I. Revisão sistemática da literatura. Bubok. (Ed.), 2010.

SAUR-AMARAL, I. Revisão sistemática da literatura com apoio de Endnote X4 e NVivo 9. Lisboa: Bubok, 2011.

SLAVIN, R. Perspectives on evidence-based research in education - what works? Issues in synthesizing educational program evaluations. Educational Researcher, 37(1), 5-14, 2008.

SQUIRES, \& ET AL. Validation of the conceptual research utilization scale: an application of the standards for educational and psychological testing in healthcare. BMC Health Services Research, 11(107), 2011.

STEWART, R., \& OLIVER, S. Making a difference with systematic reviews. In D. Gough, S. Oliver \& J. Thomas (Eds.), An Introduction to Systematic Reviews. London: Sage, 2012.

THORPE, R., HOLT, R., MACPHERSON, A., \& PITTAWAY, L.. Using knowledge within small and medium sized firms: A systematic review of the evidence. International Journal of Management Reviews, 7(4), 2005 (pp. 257-281).

TRANFIELD, D., DENYER, D., \& SMART, P. Towards a methodology for developing evidence-informed management knowledge by means of systematic review. British Journal of Management, 14(3), 2003 (pp. 207-222).

TRANFIELD, D., \& MOUCHEL, D. D. Developing an evidence-based approach to management knowledge using systematic review: Advanced Management Research Centre, Cranfield School of Management, 2002.

WARD, V., HOUSE, A., \& HAMER, S.. Developing a framework for transferring knowledge into action: a thematic analysis of the literature. Journal of Health Services Research and Policy, 14(3), 2009 (pp. 156-164). 